\title{
Improved adherence to Mediterranean Diet in adults with type 1 diabetes mellitus
}

\author{
Minerva Granado-Casas ${ }^{1,7} \cdot$ Nuria Alcubierre ${ }^{7} \cdot$ Mariona Martín $^{4} \cdot$ Jordi Real ${ }^{1,2,3}$ • Anna M. Ramírez-Morros ${ }^{1}$. \\ Maribel Cuadrado ${ }^{4}$. Núria Alonso ${ }^{1,4,8} \cdot$ Mireia Falguera $^{6} \cdot$ Marta Hernández $^{5,7} \cdot$ Eva Aguilera $^{1}$ - Albert Lecube ${ }^{5,7}$. \\ Esmeralda Castelblanco ${ }^{1,8} \cdot$ Manel Puig-Domingo ${ }^{1,8} \cdot$ Dídac Mauricio $^{1,7,8}$
}

Received: 10 January 2018 / Accepted: 11 July 2018 / Published online: 17 July 2018

(c) The Author(s) 2018

\begin{abstract}
Purpose We aimed to assess food intake and adherence to the Mediterranean Diet in patients with T1D compared with nondiabetic individuals.

Methods This was an observational, multicenter study in 262 T1D subjects and 254 age- and sex-matched nondiabetic subjects. A validated food-frequency questionnaire was administered. The alternate Mediterranean Diet Score (aMED) and alternate Healthy Eating Index (aHEI) were assessed. The clinical variables were also collected. The analysis of data included comparisons between groups and multivariate models.

Results Compared to the controls, the patients with T1D had a higher intake of dairy products $(p<0.001)$, processed meat $(p=0.001)$, fatty fish $(p=0.009)$, fruits and vegetables $(p<0.001)$, nuts $(p=0.011)$, legumes $(p<0.001)$, potatoes $(p=0.045)$, and bread $(p=0.045)$, and a lower intake of seafood $(p=0.011)$, sweets $(p<0.001)$, and alcohol drinks $(p=0.025)$. This intake pattern resulted in a higher consumption of complex carbohydrates $(p=0.049)$, fiber $(p<0.001)$, protein $(p<0.001)$, polyunsaturated fatty acids (PUFA) $(p=0.007)$, antioxidants $(p<0.001)$, vitamins $(p<0.001)$, and minerals $(p<0.001)$. The frequency of patients with T1D and low aMED score $(23.2 \%)$ was lower than that of the controls $(35.4 \% ; p=0.019)$. The overall multivariate analysis showed that, among other factors, being a T1D subject was associated with improved aMED and aHEI scores $(p=0.006$ and $p<0.001)$. In patients with T1D, residing in a nonurban area was associated with improved aMED and aHEI scores $(p=0.001$ and $p<0.001)$.

Conclusions Adult patients with T1D showed healthier dietary habits and a higher adherence to the Mediterranean Diet than nondiabetic subjects. Residing in a nonurban area is associated with an improved dietary pattern.
\end{abstract}

Keywords Type 1 diabetes mellitus $\cdot$ Mediterranean Diet $\cdot$ Dietary habits $\cdot$ Food intake $\cdot$ Intake pattern $\cdot$ Medical nutrition therapy

Electronic supplementary material The online version of this article (https://doi.org/10.1007/s00394-018-1777-z) contains supplementary material, which is available to authorized users.

Esmeralda Castelblanco

esmeraldacas@gmail.com

Dídac Mauricio

didacmauricio@gmail.com

1 Department of Endocrinology and Nutrition, Health Sciences Research Institute and University Hospital Germans Trias i Pujol, 08916 Badalona, Spain

2 Epidemiology and Public Health, International University of Catalonia, Barcelona, Spain

3 Unit Support of Research, Institut d'Investigació en Atenció Primària Jordi Gol (IDIAP Jordi Gol), Barcelona, Spain
4 Department of Endocrinology and Nutrition, University Hospital Germans Trias i Pujol, Badalona, Spain

5 Department of Endocrinology and Nutrition, University Hospital Arnau de Vilanova, Lleida, Spain

6 Primary Health Care Centre Igualada Nord, Consorci Sanitari de l'Anoia, Igualada, Spain

7 Biomedical Research Institute, University of Lleida, Lleida, Spain

8 Centre for Biomedical Research on Diabetes and Associated Metabolic Diseases (CIBERDEM), Instituto de Salud Carlos III, Barcelona, Spain 


\section{Introduction}

Medical nutrition therapy (MNT) and physical activity, in addition to insulin therapy, are cornerstones in the management of type 1 diabetes (T1D). MNT produces health benefits on glucose control, lipid profile, weight management, and maintenance of muscle mass [1-3]. Different scientific societies have issued nutritional recommendations for the medical nutrition management of T1D [2-5]. MNT is also an integral component of diabetes self-management education [2]. The handling of dietary carbohydrate content and insulin management are mainly targeted to enable the patients with T1D to self-manage the disease $[2,3]$; furthermore, healthy eating recommendations are usually included in the MNT, because these patients are regarded as a high cardiovascular risk group [2, 3, 5].

In our region, the dietary habits are identified by the Mediterranean Diet (MedDiet). This dietary pattern includes moderate intake of energy, low intake of animal fat, high intake of fruits, vegetables, whole grains, legumes, olive oil and moderate intake of fish, poultry, and red wine, together with regular physical activity (25-30 min every day) [3, 6, 7]. A number of epidemiological and intervention studies have demonstrated the potential benefits of the MedDiet in preventing cardiovascular diseases [6-10]. The MedDiet has been associated with a reduction of $9 \%$ overall mortality from cardiovascular diseases and all causes [7, 10].

Several studies in T1D have assessed the comparison between the dietary habits and the American Diabetes Association (ADA) or the European Association for the Study of Diabetes (EASD) nutritional recommendations [11-15]. Higher intakes of total fat and saturated fatty acids (SFA) have been shown in European patients with T1D [11-14]. Furthermore, the intake of carbohydrates and fiber tends to be low in this population $[11,14,15]$, although the protein consumption is adequate according to the established guidelines $[12,13]$. The intake of micronutrients is high in children, adolescents, and adults with T1D $[11,15]$. The EURODIAB Study reported a positive relationship between a higher intake of fat and SFA, and a low intake of carbohydrates and fiber with glycemic control and cardiovascular risk factors $[16,17]$. Most studies have been only performed in patients with T1D, and there are very few studies that compare the dietary habits between patients with T1D and a nondiabetic group [18-20]. In addition, to the best of our knowledge, there are only two studies that assessed the adherence to the MedDiet and healthy eating pattern in a small group of Canadian patients with T1D, but without a control group [21, 22]. Furthermore, there are only two studies with a case-control design that determined the dietary habits in children and adolescents with T1D [23, 24].
Because patients with T1D receive regular nutritional education, we hypothesized that they would have healthier eating habits than nondiabetic subjects. In a Mediterranean country like ours, MNT includes recommendations for adhering to a MedDiet pattern [4]. However, there are no data on the impact of real-world MNT practices on the dietary pattern of subjects with T1D in comparison with their nondiabetic counterparts. Thus, the aim of this study was to assess the dietary habits and adherence to the MedDiet in patients with T1D and their comparison to nondiabetic counterparts. In addition, we aimed to investigate the factors related to the MedDiet and food intake pattern.

\section{Materials and methods}

\section{Subjects}

This was an observational and two-center study. A sample of 513 participants was recruited: 259 patients with T1D and 254 nondiabetic subjects matched for age and sex in each location [healthcare areas of Lleida (center 1) and Barcelona (center 2)] between January 2013 and May 2015. The location of Lleida is a mixed rural and semi-urban area, and Barcelona is a fully urban area. The cases were patients diagnosed with T1D who were regularly cared for at their reference hospital (University Hospital Arnau of Vilanova and University Hospital Germans Trias i Pujol). Participation in the study was offered to outpatients with T1D in both departments until the study sample was complete. The inclusion criteria of cases were as follows: diagnosis of T1D with a duration of more than 1 year; age $>18$ years for both cases and control participants. The exclusion criteria for both groups were as follows: being a healthcare professional, participants who showed physical and cognitive deterioration as dementia, mental diseases, and known cardiovascular diseases (ischemic heart disease, cerebrovascular disease, peripheral arterial disease, and heart failure) or previous diabetic foot disease, pregnancy, and renal insufficiency (estimated glomerular filtration rate $<60 \mathrm{~mL} /$ min). Furthermore, for T1D, we excluded patients with a condition that requires additional MNT measures, i.e., macroalbuminuria (urine albumin/creatinine ratio $>299 \mathrm{mg} / \mathrm{g}$ ). The control participants were also excluded if they had an $\mathrm{HbA} 1 \mathrm{c}$ value $\geq 6.5 \%$ [25].

\section{Study design}

In center 1, from an initial sample of 170 patients with T1D who were contacted to participate in the study, 128 subjects accepted to participate, and 3 were excluded because of the exclusion criteria, resulting in a target sample of 125 (Supplemental Fig. 1). However, 3 additional subjects were 
excluded after their inclusion because of the identification of exclusion criteria, resulting in a final sample of 122 cases. The corresponding control group was identified from a population-based study, which was performed simultaneously in that center [25]. The nondiabetic subjects were randomly selected with matching for age (in strata of 5 years) and sex with cases, resulting in a final sample of 125 controls.

In center 2, a total of 160 patients with T1D were contacted; 148 agreed to participate. From this group, 11 were excluded, because they had met the exclusion criteria. The final sample was 137 patients with T1D. The control group was recruited at the same time as the cases; from a sample of 160 controls, 151 accepted and 22 were excluded because of the exclusion criteria, resulting in a final sample of 129 controls (Supplemental Fig. 1).

The study was approved by the local Ethics Committee of both centers, and written informed consent was obtained from all the participants.

\section{Clinical variables}

The clinical variables collected for each study group are shown in Table 1. Blood and urine samples were collected, and the biochemical variables were determined using the standard methods. Cardiovascular disease was excluded based on detailed anamnesis and careful review of all clinical records. The use of any medication, including any antihypertensive or lipid-lowering agents, was also recorded. Physical activity was assessed using the validated method of Bernstein et al. [26] and Cabrera de León et al. [27]. Physical activity was classified as either regular physical activity if the subject conducted any type of physical activity that requires 4 METS (The Metabolic Equivalent) minimum, such as walking or cycling, for more than $25 \mathrm{~min} /$ day or sedentary if the subject spent less than $25 \mathrm{~min} /$ day in physical activity.
Table 1 Clinical and demographic characteristics of the study group

\begin{tabular}{|c|c|c|c|}
\hline Characteristics & $\mathrm{T} 1 \mathrm{D}(n=259)$ & Control $(n=254)$ & $p^{\mathrm{a}}$ \\
\hline Age (years) & $43.7 \pm 11.2$ & $45.4 \pm 11.2$ & 0.10 \\
\hline Men (sex) & $118(45.6)$ & $122(48.0)$ & 0.64 \\
\hline Educational level & & & 0.05 \\
\hline Less than primary & $15(6.0)$ & $6(2.4)$ & \\
\hline Primary & $74(29.8)$ & $56(22.2)$ & \\
\hline Secondary & $97(39.1)$ & $116(46.0)$ & \\
\hline Graduate or higher & $62(25.0)$ & $74(29.4)$ & \\
\hline Smoking & & & 0.43 \\
\hline No & $130(50.2)$ & $112(44.1)$ & \\
\hline Yes & $70(27.0)$ & $78(30.7)$ & \\
\hline Former smoker & $59(22.8)$ & $64(25.2)$ & \\
\hline Regular physical activity & $186(71.8)$ & $130(51.2)$ & $<0.001$ \\
\hline Diabetes duration (years) & $21.5 \pm 10.5$ & - & - \\
\hline BMI $\left(\mathrm{kg} / \mathrm{m}^{2}\right)$ & $25.6 \pm 4.1$ & $25.9 \pm 4.0$ & 0.44 \\
\hline Waist circumference $(\mathrm{cm})$ & $88.3 \pm 12.7$ & $92.5 \pm 11.8$ & $<0.001$ \\
\hline Systolic blood pressure $(\mathrm{mmHg})$ & $126.6 \pm 17.4$ & $122.6 \pm 15.5$ & 0.009 \\
\hline Diastolic blood pressure $(\mathrm{mmHg})$ & $73.7 \pm 9.5$ & $77.3 \pm 9.4$ & $<0.001$ \\
\hline Antihypertensive treatment & $64(24.7)$ & $18(7.1)$ & $<0.001$ \\
\hline Dyslipidemia treatment & $102(39.4)$ & $39(15.4)$ & $<0.001$ \\
\hline HbA1c (\%) & $7.6 \pm 0.9$ & $5.5 \pm 0.4$ & $<0.001$ \\
\hline HbA1c (mmol/mol) & $59.4 \pm 10.4$ & $37.0 \pm 4.1$ & $<0.001$ \\
\hline Total cholesterol (mg/dL) & $180.6 \pm 28.5$ & $194.4 \pm 37.9$ & $<0.001$ \\
\hline HDLc (mg/dL) & $64.7 \pm 16.0$ & $61.4 \pm 16.0$ & 0.025 \\
\hline LDLc (mg/dL) & $101.9 \pm 23.4$ & $115.9 \pm 33.5$ & $<0.001$ \\
\hline Triglycerides (mg/dL) & $73.7 \pm 37.8$ & $94.3 \pm 53.2$ & $<0.001$ \\
\hline
\end{tabular}

Values are mean $\pm \mathrm{SD}$ or $n(\%)$

$B M I$ body mass index, $H b A 1 c$ glycated hemoglobin, $H D L c$ high-density lipoprotein cholesterol, $L D L c$ lowdensity lipoprotein cholesterol, T1D type 1 diabetes

${ }^{\mathrm{a}} p$ was calculated according to the method of Benjamini and Hochberg 


\section{Assessment of food pattern intake and adherence to dietary index}

The Food-Frequency Questionnaire Consumption (FFQC) was administered by personal interview by specialized and trained researchers. This is a semiquantitative questionnaire, validated and adapted for the Spanish population and based on the Nurses' Health Study [28, 29]. The questionnaire contains 101 items that ask each individual about his or her usual consumption over the previous year's visit. To assess the degree of adherence to the MedDiet, we used the alternate Mediterranean Diet Score (aMED) based on the Mediterranean Diet scale [30]. We also determined the alternate Healthy Eating Index (aHEI) based on the Dietary Guidelines for Americans and the Food Guide Pyramid [31]. The aMED includes vegetables, legumes, fruits, nuts, grains (only whole grain), red and processed meat, fish, monounsaturated fatty acids (MUFA)-to-SFA ratio, and alcohol intake. The score ranges from 0 (minimal adherence) to 9 (maximal adherence). The aHEI includes vegetables, fruit, nuts and soy, white and red meats, cereal fiber, trans fat, polyunsaturated and saturated fat, and alcohol intake. Energy and nutrient intake was obtained according to standardized measurement units. Daily nutrient intake was calculated adjusting for energy intake and expressed as units/ day according to the standardized methods for the FFQC.

\section{Sample size}

The sample size was calculated to detect the differences between the study groups based on previous results from a pilot study performed to assess the potential output (60 cases and 60 controls) in which the mean and standard deviation values of aMED were estimated in both groups: T1D $4.2 \pm 1.4$ and controls $3.7 \pm 1.4$. Based on this difference, using a significance level of $5 \%(\alpha=0.05)$ and a statistical power of $80 \%(\beta=0.2)$, a sample of 125 cases and 125 controls was necessary to detect a statistically significant difference in the aMED score. Finally, based on the availability of a sufficient number of potential participants, we aimed to conduct the study in two different geographical areas, which also allowed us to increase the statistical power of the study.

\section{Data analyses}

An initial descriptive comparison between groups of all variables was performed. The quantitative variables were summarized using the mean and standard deviation (SD) values, and the qualitative variables were summarized using the absolute $(n)$ and relative frequencies (\%). Statistical significance was assessed using the Chi-squared test to assess the differences in the frequencies. The mean of clinical determinations, daily food and nutrient intake, and the dietary quality index were compared using Student's $t$ test. The "BH" (aka "fdr") and "BY" methods of Benjamini, Hochberg, and Yekutieli were performed; they control the false discovery rate and the expected proportion of false discoveries among the rejected hypotheses. The false discovery rate is a less stringent condition than the family-wise error rate, so these methods are more powerful than the others. The multivariate regression models were developed to analyze the relationship between group (case and control) and dietary quality index adjusted by the potential confounders. The models were adjusted by variables that were statistically significant in the bivariate analysis or were clinically associated with diabetes. The conditional logistic regression models were designed to explain low aMED adherence (aMED low: 0-2) and adjusting linear regression models were performed for aHEI. The goodness-of-fit assumption using the Hosmer-Lemeshow test for logistic models was assessed, and for the linear regression model, the Kolmogorov-Smirnov test was performed. The estimate measures were the odds ratio (OR) with $95 \%$ confidence interval $(95 \%$ $\mathrm{CI})$ and with logistic regression models, and the effect $(\beta)$ and standard error $( \pm S E)$ with linear regression. In all the tests, a $p<0.05$ was considered to be statistically significant.

\section{Results}

The clinical and demographic characteristics of the study groups are shown in Table 1. The patients with T1D showed lower waist circumference $(p<0.001)$ than the control group. However, the patients with T1D performed regular physical activity more frequently $(p<0.001)$, and had a high frequency of treatment for hypertension $(p<0.001)$ and dyslipidemia $(p<0.001)$. Furthermore, the patients with T1D showed a better lipid profile.

\section{Dietary habits}

The patients with T1D had a healthier food intake pattern than the control group, as shown in Table 2. Compared to the controls, the subjects with T1D had a higher intake of dairy products $(p<0.001)$, processed meat $(p=0.001)$, fatty fish $(p=0.009)$, fruits and vegetables $(p<0.001)$, nuts $(p=0.011)$, legumes $(p<0.001)$, potatoes $(p=0.045)$ and bread $(p=0.045)$, and a lower intake of seafood $(p=0.011)$, sweets $(p<0.001)$ and alcohol drinks $(p=0.025)$.

Consistent with this food pattern, there was also a differential intake of nutrients (Supplemental Table 1). The patients with T1D showed a higher intake of complex carbohydrates $(p=0.049)$, soluble and insoluble fiber $(p<0.001)$, protein $(p<0.001)$, and PUFA $(p=0.007)$; the latter included intake of omega $3(p<0.001)$, omega $6(p=0.016)$, linoleic acid $(p=0.016), \alpha$-linolenic acid $(p<0.001)$, 
Table 2 Dietary quality index and daily food intake of the study groups

\begin{tabular}{|c|c|c|c|}
\hline Items & $\mathrm{T} 1 \mathrm{D}(n=259)$ & Control $(n=254)$ & $p^{\mathrm{a}}$ \\
\hline \multicolumn{4}{|l|}{ Dietary quality index } \\
\hline aMED & $3.7 \pm 1.6$ & $3.2 \pm 1.8$ & 0.009 \\
\hline aMED & & & 0.019 \\
\hline Low $(0-2)$ & $60(23.2)$ & $90(35.4)$ & \\
\hline Moderate (3-5) & $167(64.5)$ & $139(54.7)$ & \\
\hline High (6-9) & $32(12.4)$ & $25(9.8)$ & \\
\hline aHEI & $40.7 \pm 6.5$ & $37.6 \pm 6.2$ & $<0.001$ \\
\hline aHEI & & & 0.011 \\
\hline Low $(<45)$ & $196(75.7)$ & $218(85.8)$ & \\
\hline $\operatorname{High}(\geq 45)$ & $63(24.3)$ & $36(14.2)$ & \\
\hline \multicolumn{4}{|l|}{ Daily food intake (g/day) } \\
\hline Dairy products & $401.6 \pm 240.8$ & $309.6 \pm 195.0$ & $<0.001$ \\
\hline Eggs & $20.3 \pm 11.9$ & $20.6 \pm 11.0$ & 0.82 \\
\hline White meat & $36.1 \pm 22.0$ & $35.9 \pm 21.1$ & 0.93 \\
\hline Red meat & $51.9 \pm 38.7$ & $55.7 \pm 37.9$ & 0.35 \\
\hline Processed meat & $43.6 \pm 32.2$ & $34.5 \pm 25.3$ & 0.001 \\
\hline Meat & $129.1 \pm 55.1$ & $125.0 \pm 52.7$ & 0.48 \\
\hline Lean fish & $31.4 \pm 36.5$ & $30.2 \pm 21.2$ & 0.72 \\
\hline Fatty fish & $36.2 \pm 29.3$ & $29.2 \pm 23.2$ & 0.009 \\
\hline Seafood & $9.6 \pm 8.5$ & $12.3 \pm 12.2$ & 0.011 \\
\hline Fish & $77.5 \pm 48.9$ & $70.6 \pm 37.8$ & 0.13 \\
\hline Fruits and vegetables & $490.1 \pm 233.9$ & $372.0 \pm 163.4$ & $<0.001$ \\
\hline Nuts & $14.7 \pm 29.3$ & $9.0 \pm 14.0$ & 0.011 \\
\hline Legumes & $34.2 \pm 25.1$ & $25.9 \pm 19.7$ & $<0.001$ \\
\hline Cereals and pasta & $82.8 \pm 44.5$ & $77.7 \pm 42.6$ & 0.29 \\
\hline Potatoes & $55.7 \pm 39.6$ & $48.1 \pm 37.4$ & 0.045 \\
\hline Bread & $104.5 \pm 52.6$ & $93.8 \pm 56.1$ & 0.045 \\
\hline Sweets & $17.4 \pm 20.9$ & $38.5 \pm 35.1$ & $<0.001$ \\
\hline Vegetable fats & $40.0 \pm 16.7$ & $38.8 \pm 19.6$ & 0.52 \\
\hline Animal fats & $0.2 \pm 1.0$ & $0.2 \pm 0.7$ & 0.72 \\
\hline Alcohol drinks & $92.5 \pm 168.8$ & $139.0 \pm 244.5$ & 0.025 \\
\hline $\begin{array}{l}\text { Non-alcoholic bever- } \\
\text { ages }\end{array}$ & $1504.1 \pm 549.8$ & $1447.8 \pm 467.4$ & 0.29 \\
\hline Coffee and tea & $421.7 \pm 280.4$ & $448.0 \pm 312.3$ & 0.40 \\
\hline Prepared meals & $69.2 \pm 83.8$ & $61.3 \pm 54.7$ & 0.29 \\
\hline Salt & $1.1 \pm 1.8$ & $0.9 \pm 1.1$ & 0.19 \\
\hline
\end{tabular}

Values are mean \pm SD or $n(\%)$

$T 1 D$ type 1 diabetes, $a M E D$ alternate Mediterranean Diet index, aHEI alternate Healthy Eating Index

${ }^{\mathrm{a}} p$ was calculated according to the method of Benjamini and Hochberg

eicosapentaenoic acid (EPA) $(p=0.049)$, and docosahexaenoic acid (DHA) $(p=0.016)$. Furthermore, subjects with T1D also showed a higher consumption of all vitamins $(p<0.001$; except vitamin B12), carotenoids $(p<0.001)$, and minerals $(p<0.001$; except for iron intake). In contrast, the control group showed a higher intake of energy $(p=0.011)$, sugar $(p<0.001)$, stearic acid $(p=0.021)$, and alcohol $(p=0.002)$; they also had a higher dietary glycemic index $(p=0.002)$.

\section{Alternate Mediterranean Diet Score}

The dietary quality index of each of the study groups is shown in Table 2. Compared to the control group, the participants with T1D showed a higher aMED score $(p=0.009)$. Furthermore, the frequency of patients with T1D with poor adherence to a MedDiet (low aMED score) was lower $(p=0.019)$. However, both groups had a moderate mean aMED score $(3.7 \pm 1.6, \mathrm{~T} 1 \mathrm{D} ; 3.2 \pm 1.8$, control). The multivariate logistic analysis showed that being a subject with T1D $(p=0.006)$, increased physical activity $(p=0.017)$ and increasing age $(p=0.008)$ were negatively related to a low aMED score; thus, all these variables were associated with a higher adherence to a MedDiet (Fig. 1a, Supplemental Table 2).

\section{Alternate Healthy Eating Index}

Patients with T1D also showed a higher aHEI score than the controls $(p<0.001)$ (Table 2$)$. In addition, consistent with the results of aMED score, the frequency of patients with T1D and with a low aHEI score (defined as aHEI $<45$ points) was lower $(75.7 \%$, T1D vs. $85.8 \%$, controls; $p=0.011)$. Moreover, the mean of aHEI was relatively low in both groups $(40.7 \pm 6.5, \mathrm{~T} 1 \mathrm{D} ; 37.6 \pm 6.2$, controls). The multivariate linear analysis revealed that the factors associated with higher aHEI scores were the T1D group $(p<0.001)$, high educational level $(p=0.016)$, residing in the region of Lleida $(p<0.001)$, increased physical activity $(p=0.033)$, and increasing age $(p=0.006)$ (Fig. 1b, Supplemental Table 2). However, the male sex showed a negative association with this index $(p=0.001)$.

\section{Factors associated with the dietary pattern in T1D}

The multivariate analyses within the T1D group showed that the factor related to a high adherence to the MedDiet was residence in the region of Lleida (rural/semi-urban area) $(p=0.001)$ (Fig. 2a, Supplemental Table 3). The factors related to aHEI score were residence in Lleida $(p<0.001)$, high educational level $(p=0.025)$, and age $(p=0.006)$. The male sex was negatively associated with aHEI $(p=0.008)$ (Fig. 2b, Supplemental Table 3).

\section{Discussion}

In this study, we observed healthier dietary habits in patients with T1D in comparison with their nondiabetic counterparts. This finding resulted in a higher adherence to the MedDiet 

for the alternate Mediterranean Diet Score (aMED) and alternate Healthy Eating Index (aHEI) of the study groups. a Multivariate logistic regression for the aMED low group ( $0-2$ points). Hosmer-Lemeshow test $p$ value: 0.08 . b Multivariate linear regression for the aHEI. Multiple $R^{2}$ : 0.16; adjusted $R^{2}$ : 0.14 . T1D, type 1 diabetes
Fig. 1 Multivariate analysis
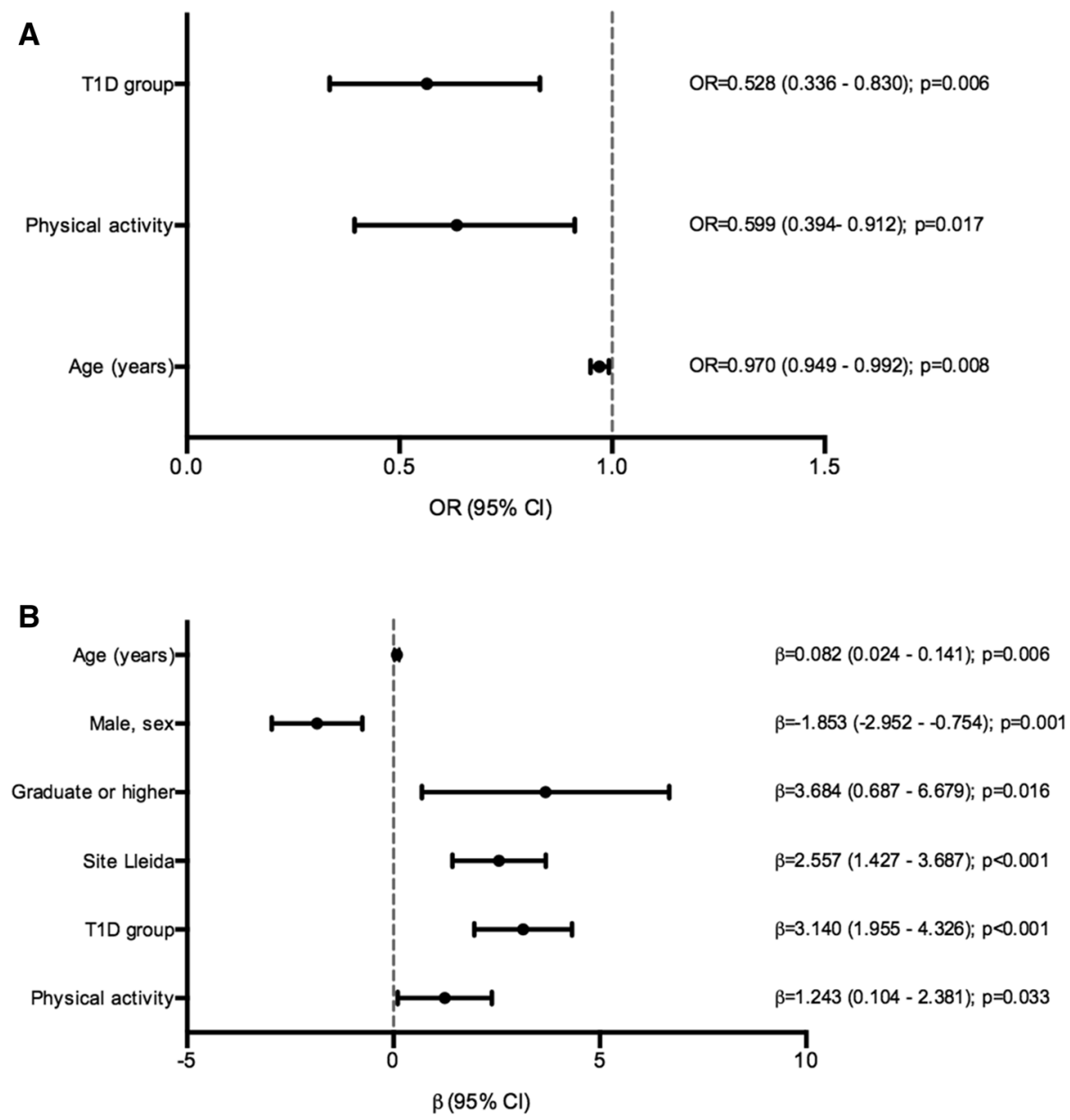

and healthier eating patterns, with higher aMED and aHEI scores in patients with T1D. In addition, we found a positive relationship between T1D, physical activity, and age with both dietary scores. The participants who were residing in the rural/semi-urban area and had a high educational level showed an increased aHEI score, whereas the male sex was negatively related to it. This is the first study that assesses food and nutrient intake, adherence to the MedDiet, and a healthy eating pattern in adult patients with T1D. Moreover, this study was specifically designed to address this question. To the best of our knowledge, no study has addressed the issue of the adherence to the MedDiet between adult subjects with T1D and a nondiabetic group.

Consistent with the previous findings [11-15], despite patients with T1D showing a moderate aMED and healthier dietary pattern, the adherence to the nutritional recommendations cannot be regarded as optimal in terms of the recommendations [2-5]. There are only three studies that compared the dietary habits between patients with T1D and nondiabetic subjects [18-20]. Snell-Bergeon et al. [18] and Jaacks et al. [19] observed a lower energy intake and higher intakes of protein and vegetables in patients with T1D; this observation is concordant with the current findings.
However, they showed a low intake of carbohydrates, high intake of fat derived from SFAs, and similar glycemic index, which is in contrast with our results. In addition, an audit of dietary management in adults with T1D found no differences in terms of macronutrient intake between the patients with T1D and a control group, although there was a potenage and sex [20]. Actually, there are only two studies using a case-control design that showed healthier dietary habits in children and adolescents with T1D [23, 24]. However, it is conceivable that the dietary patterns of children and adolescents with T1D are mainly determined by the parental dietary habits $[23,24]$. Therefore, ours is the first study showing healthier dietary patterns in adult patients with T1D and a matched nondiabetic group.

In the current study, patients with T1D had more frequently higher aMED and aHEI scores in comparison with a control group. Nevertheless, the mean of aHEI was low in both groups according to the index classification. To date, there is no study that has assessed the adherence to the MedDiet and healthy eating using this study design. Only a Canadian study has shown a moderate adherence to the MedDiet with less than half of the patients with T1D (49\%) showing tial selection bias, and both groups were not matched for 
Fig. 2 Multivariate analysis for the alternate Mediterranean Diet Score (aMED) and alternate Healthy Eating Index (aHEI) of the type 1 diabetes group. a Multivariate logistic regression for the aMED low group ( $0-2$ points). Hosmer-Lemeshow test $p$ value: 0.163 . b Multivariate linear regression for the aHEI. Multiple $R^{2}$ : 0.20; adjusted $R^{2}$ : 0.15
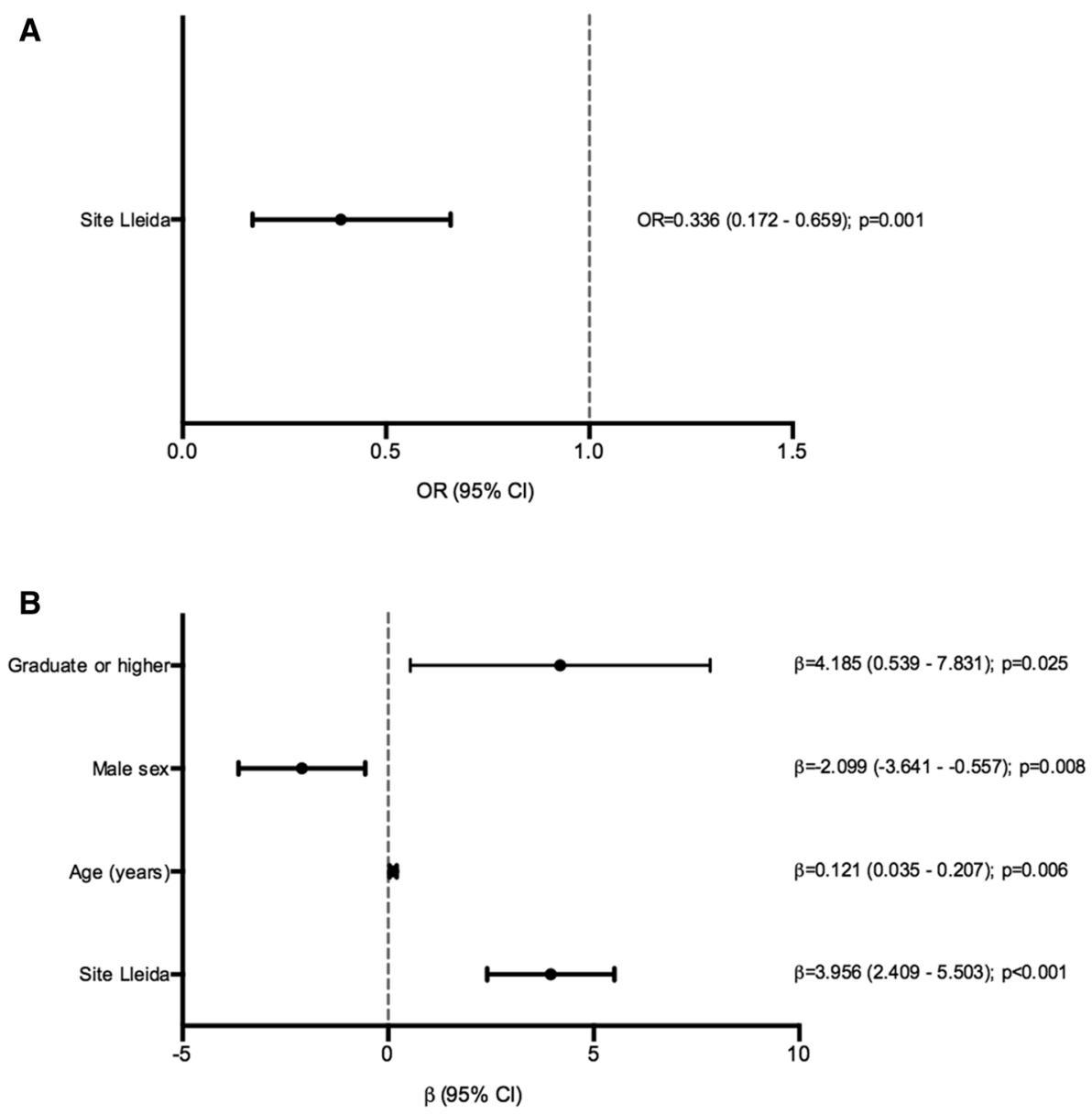

a high Canadian Healthy Eating Index (C-HEI); this finding is similar to our results [21,22]. However, that study was performed using a small sample without a nondiabetic control group.

We must underscore that the fact of not residing in an urban area was the factor that was closely and consistently associated with improved dietary quality indexes in patients with T1D. This points to a relevant influence of the sociogeographical context in determining the dietary pattern of any given subject. In addition, discordant results found by different studies in T1D may, in part, be attributed to this factor. This is also an important finding for future studies, as the control group should be carefully selected.

In our study, the subjects with a high educational level, regular physical activity, and older age showed an improved healthy lifestyle behavior independent of the disease duration. It is reasonable to think that a healthy lifestyle is more likely to be adopted in older patients and that it is maintained over time [32]. In addition, as in other studies, men show an unhealthier dietary pattern than women [22, 33]. We did not find a relationship between aMED and aHEI with the presence of dyslipidemia or hypertension, although an inverse association between lipid profile, blood pressure, and a healthy lifestyle has been reported in patients with T1D [22]. Therefore, in our population, dyslipidemia and hypertension do not seem to be potential factors that enhance the adherence to a healthier dietary pattern.

The current study has several limitations. No relationship of direct causality can be established between the different variables associated with the MedDiet, because the changes in lifestyle habits produced over time cannot be addressed with a study like the current one. Although the current results point to the probable influence on the better dietary habits of T1D patients of the educational dietary intervention (usually mainly focused on carbohydrate counting) that they receive from the treating healthcare professionals, we cannot conclude that this is the case. Unfortunately, we did not assess the knowledge of patients about the dietary management of diabetes. This study has several strengths. The large number of participants, the multicenter design, and a well-characterized sample allow us to establish the variability of different populations and lifestyles associated with them. Furthermore, we could study the different pattern in two different areas of the same region, North-Eastern Spain, that differed by their urban and rural/semi-urban locations. Moreover, this is the first study that assessed the adherence 
to the MedDiet between adults with T1D and a nondiabetic control group.

In conclusion, in our Mediterranean region, adults with T1D showed a healthier food intake pattern and, specifically, an improved adherence to the MedDiet than their nondiabetic counterparts. However, additional research is warranted in this field and in the identification of the educational strategies that need to be adopted to enhance the adherence of patients with T1D to a healthy diet.

Acknowledgements This study was supported by the Catalan Diabetes Association (Beca d'Educació Terapèutica 2015), Spain. Additional support from Grants PI12/00183 and PI15/00625 from the Instituto de Salud Carlos III (Ministry of Economy and Competitiveness, Spain) to DM is acknowledged. CIBERDEM is an initiative from the Instituto de Salud Carlos III (Plan Nacional de I + D + I and Fondo Europeo de Desarrollo Regional). MG-C holds a predoctoral fellowship from the Ministerio de Educación, Cultura y Deporte, FPU15/03005.

Author contributions The authors' responsibilities were as follows: MG-C, NA, and DM designed the research; MG-C, AR-M, NA, MM, EC, MF, MH, MC, and EA conducted research; MG-C, JR, and DM analyzed the data; MG-C, NA, JR, and DM wrote the paper; AL and MP-D coordinated the research; DM was the principal investigator and had the primary responsibility for the final content. All the authors read and approved the final manuscript. MG-C and NA contributed equally to this work.

\section{Compliance with ethical standards}

Conflict of interest The authors declare that they have no conflict of interest.

Open Access This article is distributed under the terms of the Creative Commons Attribution 4.0 International License (http://creativeco mmons.org/licenses/by/4.0/), which permits unrestricted use, distribution, and reproduction in any medium, provided you give appropriate credit to the original author(s) and the source, provide a link to the Creative Commons license, and indicate if changes were made.

\section{References}

1. Mann JI (1997) The role of nutritional modifications in the prevention of macrovascular complications of diabetes. Diabetes 46(Suppl 2):S125-S130. https://doi.org/10.2337/diab.46.2.S125

2. Bantle JP, Wylie-Rosett J, Albright AL, Apovian CM, Clark NG, Franz MJ, Hoogwerf BJ, Lichtenstein AH, Mayer-Davis E, Mooradian AD, Wheeler ML (2008) Nutrition recommendations and interventions for diabetes: a position statement of the American Diabetes Association. Diabetes Care 31:S61-S78. https://doi. org/10.2337/dc08-S061

3. Mann J, Lean M, Toeller M (2000) Recommendations for the nutritional management of patients with diabetes mellitus. Eur J Clin Nutr 54:353-355

4. Catalan Association of Diabetes (2013) Documento de consenso sobre "Recomendaciones nutricionales y de educación alimentaria en la diabetes. ACD, Barcelona
5. American Diabetes Association (2017) Lifestyle management. Section 4. In Standards of Medical Care in Diabetes-2017. Diabetes Care 40:S33-S43. https://doi.org/10.2337/dc17-S007

6. Estruch R, Ros E, Salas-Salvadó J, Covas M-I, Corella D, Arós F, Gómez-Gracia E, Ruiz-Gutiérrez V, Fiol M, Lapetra J, LamuelaRaventos RM, Serra-Majem L, Pintó X, Basora J, Muñoz MA, Sorlí JV, Martínez JA, Martínez-González MA (2013) Primary prevention of cardiovascular disease with a Mediterranean diet. N Engl J Med 368:1279-1290. https://doi.org/10.1056/NEJMo a1200303

7. Sofi F, Cesari F, Abbate R, Gensini GF, Casini A (2008) Adherence to Mediterranean diet and health status: meta-analysis. BMJ 337:a1344. https://doi.org/10.1136/bmj.a1344

8. Estruch R, Martínez-González MA, Corella D, Salas-Salvadó J, Ruiz-Gutiérrez V, Covas MI, Fiol M, Gómez-Gracia E, LópezSabater MC, Vinyoles E, Arós F, Conde M, Lahoz C, Lapetra J, Sáez G, Ros E (2006) Effects of a Mediterranean-style diet on cardiovascular risk factors: a randomized trial. Ann Intern Med 145:1-11. https://doi.org/10.7326/0003-4819-145-1-20060 7040-00004

9. Esposito K, Marfella R, Ciotola M, Di Palo C, Giugliano F, Giugliano G, D’Armiento M, D'Andrea F, Giugliano D (2004) Effect of a mediterranean-style diet on endothelial dysfunction and markers of vascular inflammation in the metabolic syndrome: a randomized trial. JAMA 292:1440-1446. https://doi. org/10.1016/j.accreview.2004.11.073

10. Lairon D (2007) Intervention studies on Mediterranean diet and cardiovascular risk. Mol Nutr Food Res 51:1209-1214. https:// doi.org/10.1002/mnfr.200700097

11. Ahola AJ, Mikkilä V, Mäkimattila S, Forsblom C, Freese R, Groop P-H, FinnDiane Study Group (2012) Energy and nutrient intakes and adherence to dietary guidelines among Finnish adults with type 1 diabetes. Ann Med 44:73-81. https://doi. org/10.3109/07853890.2010.530682

12. Soedamah-Muthu SS, Chaturvedi N, Fuller JH, Toeller M (2013) Do European people with type 1 diabetes consume a high atherogenic diet? 7-year follow-up of the EURODIAB Prospective Complications Study. Eur J Nutr 52:1701-1710. https ://doi.org/10.1007/s00394-012-0473-7

13. The Diabetes and Nutrition Study Group of the Spanish Diabetes Association (2006) Diabetes Nutrition and Complications Trial: adherence to the ADA nutritional recommendations, targets of metabolic control, and onset of diabetes complications. A 7-year, prospective, population-based, observational multicenter study. J Diabetes Complicat 20:361-366. https:// doi.org/10.1016/j.jdiacomp.2005.09.003

14. The Diabetes and Nutrition Study Group of the Spanish Association (2004) Diabetes Nutrition and Complications Trial. Trends in nutritional pattern between 1993 and 2000 and targets of diabetes treatment in a sample of Spanish people with diabetes. Diabetes Care 27:2000-2003

15. Mayer-Davis EJ, Nichols M, Liese AD, Bell RA, Dabelea DM, Johansen JM, Pihoker C, Rodriguez BL, Thomas J, Williams D (2006) Dietary intake among youth with diabetes: the SEARCH for diabetes in Youth Study. J Am Diet Assoc 106:689-697. https://doi.org/10.1016/j.jada.2006.02.002

16. Balk S, Schoenaker D, Mishra G, Toeller M, Chaturvedi N, Fuller J, Soedamah-Muthu S (2015) Association of diet and lifestyle with glycated haemoglobin in type 1 diabetes participants in the EURODIAB prospective complications study. Eur J Clin Nutr 70:229-236. https://doi.org/10.1038/ejcn.2015.110

17. Toeller M, Buyken a E, Heitkamp G, Cathelineau G, Ferriss B, Michel G (2001) Nutrient intakes as predictors of body weight in European people with type 1 diabetes. Int J Obes Relat Metab Disord 25:1815-1822. https://doi.org/10.1038/sj.ijo.0801816 
18. Snell-Bergeon JK, Chartier-Logan C, Maahs DM, Ogden LG, Hokanson JE, Kinney GL, Eckel RH, Ehrlich J, Rewers M (2009) Adults with type 1 diabetes eat a high-fat atherogenic diet that is associated with coronary artery calcium. Diabetologia 52:801-809. https://doi.org/10.1007/s00125-009-1280-4

19. Jaacks LM, Du S, Mendez MA, Crandell J, Liu W, Ji L, Rosamond W, Popkin BM, Mayer-Davis EJ (2015) Comparison of the dietary intakes of individuals with and without type 1 diabetes in China. Asia Pac J Clin Nutr 24:639-649. https://doi. org/10.6133/apjen.2015.24.4.03

20. Tahbaz F, Kreis I, Calvert D (2006) An audit of diabetes control, dietary management and quality of life in adults with type 1 diabetes mellitus, and a comparison with nondiabetic subjects. J Hum Nutr Diet 19:3-11. https://doi.org/10.1111/j.1365277X.2006.00668.x

21. Gingras V, Leroux C, Desjardins K, Savard V, Lemieux S, RabasaLhoret R, Strychar I (2015) Association between cardiometabolic profile and dietary characteristics among adults with type 1 diabetes mellitus. J Acad Nutr Diet 115:1965-1974. https://doi. org/10.1016/j.jand.2015.04.012

22. Leroux C, Gingras V, Desjardins K, Brazeau AS, Ott-Braschi S, Strychar I, Rabasa-Lhoret R (2015) In adult patients with type 1 diabetes healthy lifestyle associates with a better cardiometabolic profile. Nutr Metab Cardiovasc Dis 25:444-451. https://doi. org/10.1016/j.numecd.2015.01.004

23. Maffeis C, Morandi A, Ventura E, Sabbion A, Contreas G, Tomasselli F, Tommasi M, Fasan I, Costantini S, Pinelli L (2012) Diet, physical, and biochemical characteristics of children and adolescents with type 1 diabetes: relationship between dietary fat and glucose control. Pediatr Diabetes 13:137-146. https://doi.org/10. 1111/j.1399-5448.2011.00781.x

24. Lodefalk M, Aman J (2006) Food habits, energy and nutrient intake in adolescents with type 1 diabetes mellitus. Diabet Med 23:1225-1232. https://doi.org/10.1111/j.1464-5491.2006.01971 .x

25. Vilanova MB, Falguera M, Marsal JR, Rubinat E, Alcubierre N, Catelblanco E, Granado-Casas M, Miró N, Molló À, Mata-Cases M, Franch-Nadal J, Mauricio D (2017) Prevalence, clinical features and risk assessment of pre-diabetes in Spain: the prospective Mollerussa cohort study. BMJ Open 7:e015158. https://doi. org/10.1136/bmjopen-2016-015158
26. Bernstein MS, Morabia A, Sloutskis D (1999) Definition and prevalence of sedentarism in an urban population. Am J Public Health 89:862-867. https://doi.org/10.2105/AJPH.89.6.862

27. Cabrera de León A, Rodríguez-Pérez MDC, Rodríguez-Benjumeda LM, Anía-Lafuente B, Brito-Díaz B, Muros de Fuentes M, Almeida-González D, Batista-Medina M, Aguirre-Jaime A (2007) Sedentary lifestyle: physical activity duration versus percentage of energy expenditure. Rev Esp Cardiol 60:244-250. https://doi. org/10.1016/S1885-5857(07)60148-0

28. Willett WC, Sampson L, Stampfer MJ, Rosner B, Bain C, Witschi J, Hennekens CH, Speizer FE (1985) Reproducibility and validity of a semiquantitative food frequency questionnaire. Am J Epidemiol 122:51-65. https://doi.org/10.1093/acprof

29. Vioque J, Navarrete-Muñoz E-M, Gimenez-Monzó D, Garcíade-la-Hera M, Granado F, Young IS, Ramón R, Ballester F, Murcia M, Rebagliato M, Iñiguez C (2013) Reproducibility and validity of a food frequency questionnaire among pregnant women in a Mediterranean area. Nutr J 12:26. https://doi. org/10.1186/1475-2891-12-26

30. Trichopoulou A, Costacou T, Bamia C, Trichopoulos D (2003) Adherence to a Mediterranean diet and survival in a Greek population. N Engl J Med 348:2599. https://doi.org/10.1056/NEJMo a1005372

31. Fung TT, McCullough ML, Newby PK, Manson JE, Meigs JB, Rifai N, Willett WC, Hu FB (2005) Diet-quality scores and plasma concentrations of markers of inflammation and endothelial dysfunction. Am J Clin Nutr 82:163-173

32. Zamora-Ros R, Knaze V, Luján-Barroso L, Romieu I, Scalbert A, Slimani N, Hjartåker A, Engeset D, Skeie G, Overvad K, Bredsdorff L, Tjønneland A, Halkjær J, Key TJ, Khaw K-T, Mulligan AA, Winkvist A, ...González CA (2013) Differences in dietary intakes, food sources and determinants of total flavonoids between Mediterranean and non-Mediterranean countries participating in the European Prospective Investigation into Cancer and Nutrition (EPIC) study. Br J Nutr 109:1498-1507. https://doi.org/10.1017/ S0007114512003273

33. Bondia-Pons I, Serra-Majem L, Castellote AI, Lopez-Sabater MC (2007) Identification of foods contributing to the dietary lipid profile of a Mediterranean population. Br J Nutr 98:583-592. https:// doi.org/10.1017/S0007114507727435 